Cultures \& Conflits

45 | printemps 2002

De Tampere à Séville : bilan de la sécurité européenne $(1 / 2)$

\title{
Conseil européen de Tampere 15 et 16 Octobre 1999
}

\section{Union européenne}

\section{(2) OpenEdition \\ Journals}

Édition électronique

URL : http://journals.openedition.org/conflits/777

DOI : $10.4000 /$ conflits. 777

ISSN : $1777-5345$

Éditeur :

CCLS - Centre d'études sur les conflits lilberté et sécurité, L'Harmattan

Édition imprimée

Date de publication : 1 mars 2002

ISBN : 2-7475-3029-9

ISSN : 1157-996X

\section{Référence électronique}

Union européenne, «Conseil européen de Tampere 15 et 16 Octobre 1999 », Cultures \& Conflits [En ligne], 45 I printemps 2002, mis en ligne le 22 mars 2006, consulté le 30 mars 2021. URL : http:// journals.openedition.org/conflits/777 ; DOI : https://doi.org/10.4000/conflits. 777

Ce document a été généré automatiquement le 30 mars 2021.

Creative Commons License 


\title{
Conseil européen de Tampere 15 et 16 Octobre 1999
}

\author{
Union européenne
}

Le Conseil européen a tenu à Tampere, les 15 et 16 octobre 1999, une réunion spéciale consacrée à la création d'un espace de liberté, de sécurité et de justice dans l'Union européenne. Les travaux ont débuté par un échange de vues avec Mme Nicole Fontaine, Présidente du Parlement européen, sur les principaux thèmes de discussion.

3 Le Conseil européen est déterminé à faire de l'Union un espace de liberté, de sécurité et de justice en exploitant pleinement les possibilités offertes par le traité d'Amsterdam. Le Conseil européen réaffirme l'importance de cet objectif par un message politique fort et il a marqué son accord sur un certain nombre d'orientations et de priorités politiques qui feront rapidement de cet espace une réalité.

Le Conseil européen entend placer cet objectif en tête de l'agenda politique et l'y maintenir. Il examinera régulièrement les progrès réalisés dans la mise en œuvre des mesures nécessaires et le respect des échéances fixées par le traité d'Amsterdam, le plan d'action de Vienne et les présentes conclusions. La Commission est invitée à présenter une proposition de tableau de bord à cette fin. Le Conseil européen souligne qu'il importe de veiller à la transparence nécessaire et de tenir le Parlement européen régulièrement informé. Il tiendra un vaste débat pour évaluer les progrès accomplis lors de sa réunion de décembre 2001.

Dans un domaine étroitement lié à la création d'un espace de liberté, de sécurité et de justice, le Conseil européen est parvenu à un accord sur la composition, la méthode de travail et les modalités pratiques (figurant en annexe aux présentes conclusions) de l'enceinte chargée de l'élaboration d'un projet de charte des droits fondamentaux de l'Union européenne. Il invite toutes les parties concernées à faire en sorte que les travaux d'élaboration de la charte puissent débuter rapidement.

6 Le Conseil européen exprime sa reconnaissance au Secrétaire général sortant, M. Jürgen Trumpf, pour le travail qu'il a accompli au Conseil et notamment pour la 
manière dont il a contribué au développement de l'Union après l'entrée en vigueur du traité d'Amsterdam.

7 L'un des objectifs essentiels des travaux de l'Union, dans les années à venir, devant être de renforcer la politique étrangère et de sécurité commune, notamment en développant une politique européenne de sécurité et de défense, le Conseil européen attend du nouveau Secrétaire général du Conseil et Haut Représentant pour la PESC, M. Javier Solana, qu'il apporte à cet égard une contribution majeure. M. Solana pourra compter, dans l'exercice de ses compétences, sur le plein appui du Conseil européen, conformément à l'article 18 , paragraphe 3 , du traité, afin de pouvoir s'acquitter pleinement de ses tâches. Il lui incombera notamment de coopérer avec la présidence pour veiller à ce que les délibérations et l'action en matière de politique étrangère et de sécurité soient menées avec efficacité, en vue d'assurer continuité et cohérence, compte tenu des intérêts communs de l'Union.

1. Depuis ses tout premiers débuts, l'intégration européenne est fermement ancrée dans un attachement commun à la liberté reposant sur les droits de l'homme, sur des institutions démocratiques et sur l'état de droit. Ces valeurs communes se sont avérées nécessaires pour préserver la paix et accroître la prospérité dans l'Union européenne. Elles seront également la pierre angulaire de l'élargissement de l'Union.

11 2. L'Union européenne a déjà mis en place pour ses citoyens les éléments principaux d'un espace commun de prospérité et de paix: un marché unique, une union économique et monétaire et la capacité à relever les défis politiques et économiques mondiaux. Le pari du traité d'Amsterdam est de veiller maintenant à ce qu'il soit possible de jouir de la liberté, qui comprend le droit de circuler librement dans toute l'Union, dans des conditions de sécurité et de justice accessibles à tous. C'est un projet qui répond aux préoccupations souvent exprimées par les citoyens et qui aura une incidence directe sur leur vie quotidienne.

12 3. Cette liberté ne doit toutefois pas être considérée comme une prérogative des seuls citoyens de l'Union. Son existence même agit comme un aimant, attirant du monde entier nombre de personnes privées de cette liberté qui, pour les citoyens de l'Union, va de soi. Il serait contraire aux traditions de l'Europe de refuser cette liberté à ceux qui, poussés par les circonstances, demandent légitimement accès à notre territoire. L'Union se doit donc d'élaborer des politiques communes dans les domaines de l'asile et de l'immigration, tout en tenant compte de la nécessité d'exercer aux frontières extérieures un contrôle cohérent afin de stopper l'immigration clandestine et de s'opposer à ceux qui l'organisent et commettent ainsi des infractions relevant de la criminalité internationale. Ces politiques communes doivent reposer sur des principes qui à la fois soient clairs pour nos citoyens et offrent des garanties à ceux qui cherchent protection dans l'Union européenne ou demandent accès à son territoire.

13 4. Notre objectif est une Union européenne ouverte et sûre, pleinement attachée au respect des obligations de la Convention de Genève sur les réfugiés et des autres instruments pertinents en matière de droits de l'homme, et capable de répondre aux besoins humanitaires sur la base de la solidarité. Il convient également de mettre en place une approche commune pour assurer l'intégration dans nos sociétés des ressortissants de pays tiers qui résident légalement dans l'Union. 
14 5. Le citoyen ne peut jouir de la liberté que dans un véritable espace de justice, où chacun peut s'adresser aux tribunaux et aux autorités de tous les Etats membres aussi facilement qu'il le ferait dans son propre pays. Les auteurs d'infractions ne doivent pouvoir, par aucun moyen, mettre à profit les différences entre les systèmes judiciaires des Etats membres. Les jugements et décisions doivent être respectés et exécutés dans l'ensemble de l'Union, mais il faut aussi préserver la sécurité juridique fondamentale des particuliers et des opérateurs économiques. Il convient d'arriver à une meilleure compatibilité et à une convergence accrue entre les systèmes juridiques des Etats membres.

6. Les citoyens sont en droit d'attendre de l'Union qu'elle réagisse à la menace que représente la grande criminalité pour leur liberté et les droits que leur reconnait la loi. Pour contrer ces menaces, il est nécessaire d'agir de concert, dans toute l'Union, en matière de prévention et de lutte contre la criminalité et les organisations criminelles. Une mobilisation commune des ressources policières et judiciaires est nécessaire pour veiller à ce que les auteurs d'infraction et le produit de leurs crimes ne trouvent aucun refuge dans l'Union.

16 7. L'espace de liberté, de sécurité et de justice devrait faire fond sur les principes de transparence et de contrôle démocratique. Nous devons entamer un dialogue ouvert avec la société civile sur les objectifs et principes de cet espace pour qu'ils soient mieux acceptés par les citoyens et reçoivent leur soutien. Afin que les autorités continuent à jouir de la confiance des citoyens, il faut mettre en place des normes communes relatives à l'intégrité des autorités.

17 8. Le Conseil européen estime essentiel que, dans ces domaines, l'Union acquière également une capacité d'action et soit considérée comme un partenaire important sur la scène internationale. Cela nécessite une étroite coopération avec les pays partenaires et les organisations internationales, notamment le Conseil de l'Europe, l'OSCE, l'OCDE et les Nations Unies.

9. Le Conseil européen invite le Conseil et la Commission, en étroite coopération avec le Parlement européen, à favoriser la mise en œuvre intégrale et immédiate du traité d'Amsterdam, sur la base du plan d'action de Vienne ainsi que des orientations politiques et objectifs concrets convenus lors de la présente réunion de Tampere qui figurent ci-après.

\section{A. UNE POLITIQUE EUROPEENNE COMMUNE EN MATIERE D’ASILE ET DE MIGRATION}

20 10. Il faut, pour les domaines distincts, mais étroitement liés, de l'asile et des migrations, élaborer une politique européenne commune comprenant les éléments indiqués ci-après.

21 I. Partenariat avec les pays d'origine

22 11. L'Union européenne a besoin d'une approche globale des migrations qui aborde les aspects politiques, les droits de l'homme et les questions de développement dans les pays et régions d'origine et de transit. Cela exige de lutter contre la pauvreté, d'améliorer les conditions de vie et les possibilités d'emploi, de prévenir les conflits, de consolider les Etats démocratiques et de veiller au respect des droits de l'homme, notamment les droits des minorités, des femmes et des enfants. A cet effet, l'Union et les Etats membres sont invités à contribuer, dans les limites de leurs compétences respectives en vertu des traités, à l'amélioration de la cohérence des politiques intérieures et extérieures de l'Union. Le partenariat avec les pays tiers concernés 
constituera aussi un élément déterminant du succès de cette politique, dans le but de favoriser le codéveloppement.

12. Le Conseil européen se félicite à cet égard du rapport qui a été établi par le Groupe de haut niveau "Asile et Migration" institué par le Conseil, et convient de la prorogation de son mandat et de l'établissement d'autres plans d'action. Il considère que les premiers plans d'action établis par ce groupe et approuvés par le Conseil représentent une contribution utile et il invite le Conseil et la Commission à présenter un rapport sur leur mise en œuvre lors du Conseil européen de décembre 2000. repose sur la solidarité entre les Etats membres. Le Conseil européen estime qu'il convient d'envisager de constituer, sous une forme ou sous une autre, une réserve financière destinée à la protection temporaire en cas d'afflux massifs de réfugiés. La Commission est invitée à étudier cette possibilité. relatifs au système d'identification des demandeurs d'asile (Eurodac).

II. Un régime d'asile européen commun

13. Le Conseil européen réaffirme l'importance que l'Union et ses Etats membres attachent au respect absolu du droit de demander l'asile. Il est convenu de travailler à la mise en place d'un régime d'asile européen commun, fondé sur l'application intégrale et globale de la Convention de Genève et d'assurer ainsi que nul ne sera renvoyé là où il risque à nouveau d'être persécuté, c'est-à-dire de maintenir le principe de non-refoulement.

14. Ce régime devrait comporter, à court terme, une méthode claire et opérationnelle pour déterminer l'Etat responsable de l'examen d'une demande d'asile, des normes communes pour une procédure d'asile équitable et efficace, des conditions communes minimales d'accueil des demandeurs d'asile, et le rapprochement des règles sur la reconnaissance et le contenu du statut de réfugié. Il devrait aussi être complété par des mesures relatives à des formes subsidiaires de protection offrant un statut approprié à toute personne nécessitant une telle protection. A cette fin, le Conseil est instamment invité à adopter, sur la base de propositions de la Commission, les décisions nécessaires conformément au calendrier fixé par le traité d'Amsterdam et le plan d'action de Vienne. Le Conseil européen souligne qu'il importe de consulter le HCR et d'autres organisations internationales.

15. A terme, les règles communautaires devraient déboucher sur une procédure d'asile commune et un statut uniforme, valable dans toute l'Union, pour les personnes qui se voient accorder l'asile. La Commission est invitée à élaborer une communication dans ce domaine dans un délai d'un an.

16. Le Conseil européen engage le Conseil à intensifier ses efforts en vue d'arriver, sur la question de la protection temporaire des personnes déplacées, à un accord qui 17. Le Conseil européen invite instamment le Conseil à terminer rapidement les travaux III. Traitement équitable pour les ressortissants de pays tiers

18. L'Union européenne doit assurer un traitement équitable aux ressortissants de pays tiers qui résident légalement sur le territoire de ses Etats membres. Une politique plus énergique en matière d'intégration devrait avoir pour ambition de leur offrir des droits et obligations comparables à ceux des citoyens de l'Union européenne. Cette politique devrait également favoriser la non-discrimination dans la vie économique, sociale et culturelle et mettre en place des mesures de lutte contre le racisme et la xénophobie. 

le racisme", le Conseil européen demande l'intensification de la lutte contre le racisme et la xénophobie. Les Etats membres tireront parti des meilleures pratiques et de l'expérience acquise. Il conviendra de renforcer davantage la coopération avec l'Observatoire européen des phénomènes racistes et xénophobes et le Conseil de l'Europe. En outre, la Commission est invitée à présenter le plus rapidement possible des propositions de mise en œuvre de l'article 13 du traité CE relatif à la lutte contre le racisme et la xénophobie. Pour lutter contre la discrimination de manière plus générale, les Etats membres sont encouragés à élaborer des programmes nationaux.

20. Le Conseil européen reconnaît la nécessité d'un rapprochement des législations nationales relatives aux conditions d'admission et de séjour des ressortissants de pays tiers, fondé sur une évaluation commune tant de l'évolution économique et démographique au sein de l'Union que de la situation dans les pays d'origine. Il demande à cette fin au Conseil d'arrêter rapidement des décisions sur la base de propositions de la Commission. Ces décisions devraient tenir compte non seulement de la capacité d'accueil de chaque Etat membre, mais aussi de leurs liens historiques et culturels avec les pays d'origine.

21. Le statut juridique des ressortissants de pays tiers devrait être rapproché de celui des ressortissants des Etats membres. Une personne résidant légalement dans un Etat membre pendant une période à déterminer et titulaire d'un permis de séjour de longue durée devrait se voir octroyer dans cet Etat membre un ensemble de droits uniformes aussi proches que possible de ceux dont jouissent les citoyens de l'Union européenne, par exemple le droit de résider, d'étudier, de travailler à titre de salarié ou d'indépendant, ainsi que l'application du principe de non-discrimination par rapport aux citoyens de l'Etat de résidence. Le Conseil européen fait sien l'objectif d'offrir aux ressortissants de pays tiers résidant légalement depuis longtemps dans l'Union la possibilité d'obtenir la nationalité de l'Etat membre dans lequel ils résident

22. Le Conseil européen souligne qu'il est nécessaire d'assurer, à toutes les étapes, une gestion plus efficace des flux migratoires. Il demande le lancement, en coopération étroite avec les pays d'origine et de transit, de campagnes d'information sur les possibilités réelles d'immigration légale et la prévention de toutes les formes de traite d'êtres humains. Il conviendrait de poursuivre la mise en place d'une politique commune active en matière de visas et de faux documents incluant une coopération plus étroite entre les consulats de l'UE dans les pays tiers et, le cas échéant, l'établissement de bureaux communs chargés de la délivrance des visas UE.

23. Le Conseil européen est déterminé à combattre à sa source l'immigration clandestine, notamment en s'attaquant à ceux qui se livrent à la traite des êtres humains et à l'exploitation économique des migrants. Il insiste sur l'adoption de dispositions législatives prévoyant des sanctions sévères pour cette forme grave de criminalité. Le Conseil est invité à adopter, avant la fin de l'an 2000, des dispositions législatives à cette fin, sur la base d'une proposition de la Commission. En collaboration avec Europol, les Etats membres devraient concentrer leurs efforts sur la détection et le démantèlement des filières criminelles. Les droits des victimes de ces pratiques seront garantis, une attention particulière étant accordée aux problèmes auxquels sont confrontés les femmes et les enfants.

Cultures \& Conflits, 45 | printemps 2002 
38 24. Le Conseil européen demande qu'il y ait une coopération plus étroite et une entraide technique entre les services de contrôle aux frontières des Etats membres, notamment sous forme de programmes d'échanges et de transfert de technologies, en particulier aux frontières maritimes, et que les Etats candidats soient associés sans tarder à cette coopération. Dans ce contexte, le Conseil se félicite du mémorandum d'accord intervenu entre l'Italie et la Grèce en vue de renforcer la coopération entre les deux pays dans l'Adriatique et la mer Ionienne pour lutter contre la criminalité organisée, les activités des passeurs et la traite des êtres humains.

25. A la suite de l'intégration de l'acquis de Schengen dans l'Union, les pays candidats doivent accepter intégralement cet acquis ainsi que les mesures prises en vertu de celui-ci. Le Conseil européen souligne l'importance d'un contrôle efficace aux futures frontières extérieures de l'Union effectué par des professionnels dûment formés.

26. Le Conseil européen demande que l'aide aux pays d'origine et de transit soit accrue afin de faciliter les retours volontaires et de permettre aux autorités de ces pays de renforcer leurs moyens de combattre efficacement la traite des êtres humains et de satisfaire à leurs obligations en matière de réadmission à l'égard de l'Union et des Etats membres.

27. Le traité d'Amsterdam a conféré des compétences à la Communauté dans le domaine de la réadmission. Le Conseil européen invite le Conseil à conclure des accords de réadmission ou à insérer des clauses-types dans d'autres accords conclus entre la Communauté européenne et les pays ou groupes de pays tiers concernés. Il convient également d'examiner les règles relatives à la réadmission entre Etats membres.

\section{B. UN VERITABLE ESPACE EUROPEEN DE JUSTICE}

28. Dans un véritable espace européen de justice, l'incompatibilité ou la complexité des systèmes juridiques et administratifs des Etats membres ne devraient pas empêcher ou dissuader les particuliers et les entreprises d'exercer leurs droits.

\section{Un meilleur accès à la justice en Europe}

29. Afin de faciliter l'accès à la justice, le Conseil européen invite la Commission, en coopération avec d'autres enceintes compétentes, telles que le Conseil de l'Europe, à lancer une campagne d'information et à publier des "guides de l'utilisateur" bien conçus sur la coopération judiciaire au sein de l'Union et sur les systèmes juridiques des Etats membres. Il recommande également la création d'un système d'information facile d'accès, dont l'entretien et la mise à jour seraient assurés par un réseau d'autorités nationales compétentes.

30. Le Conseil européen invite le Conseil à établir, sur la base de propositions de la Commission, des normes minimales garantissant un niveau approprié d'aide juridique pour les affaires transfrontalières dans l'ensemble de l'Union ainsi que des règles de procédure spéciales communes en vue de simplifier et d'accélérer le règlement des litiges transfrontaliers concernant les demandes de faible importance en matière civile et commerciale ainsi que les créances alimentaires, et les créances certaines. Les Etats membres devraient également mettre en place des procédures de substitution extrajudiciaires.

31. Il faudrait fixer des normes communes minimales pour les formulaires ou les documents multilingues à utiliser dans les procédures judiciaires transfrontalières dans l'ensemble de l'Union. La validité de ces documents ou formulaires serait ensuite 
reconnue par les autres Etats membres dans toutes les procédures judiciaires se déroulant dans l'Union.

32. Eu égard à la communication de la Commission, il faudrait établir des normes minimales pour la protection des victimes de la criminalité, notamment en ce qui concerne l'accès à la justice de ces victimes et leur droit à réparation, y compris au remboursement des frais de justice. En outre, des programmes nationaux devraient être mis sur pied pour financer des mesures, tant publiques que non gouvernementales, d'assistance et de protection en faveur des victimes.

jugements et le rapprochement nécessaire des législations faciliteraient la coopération entre autorités et la protection judiciaire des droits de la personne. Le Conseil européen approuve donc le principe de reconnaissance mutuelle, qui, selon lui, devrait devenir la pierre angulaire de la coopération judiciaire en matière tant civile que pénale au sein de l'Union. Le principe devrait s'appliquer tant aux jugements qu'aux autres décisions émanant des autorités judiciaires.

51 34. En matière civile, le Conseil européen invite la Commission à faire une proposition visant à réduire davantage les mesures intermédiaires qui sont encore requises pour permettre la reconnaissance et l'exécution d'une décision ou d'un jugement dans l'Etat requis. Dans un premier temps, il conviendrait de supprimer ces procédures intermédiaires pour les droits concernant des demandes de faible importance en matière civile ou commerciale et pour certains jugements concernant des litiges relevant du droit de la famille (par exemple, les créances alimentaires et les droits de visite). Ces décisions seraient automatiquement reconnues dans l'ensemble de l'Union sans procédure intermédiaire ni motifs de refus d'exécution. Ce dispositif pourrait s'accompagner de la fixation de normes minimales pour certains aspects de procédure civile.

35. En matière pénale, le Conseil européen engage les Etats membres à ratifier sans tarder les conventions d'extradition de l'UE de 1995 et 1996. Il estime que la procédure formelle d'extradition devrait être supprimée entre les Etats membres pour les personnes qui tentent d'échapper à la justice après avoir fait l'objet d'une condamnation définitive et remplacée par un simple transfèrement de ces personnes, conformément à l'article $6 \mathrm{du}$ traité UE. Il convient également d'envisager des procédures accélérées d'extradition, sans préjudice du principe du droit à un procès équitable. Le Conseil européen invite la Commission à faire des propositions à ce sujet, à la lumière de la Convention d'application de Schengen.

53 36. Le principe de reconnaissance mutuelle devrait aussi s'appliquer aux décisions précédant la phase de jugement, en particulier à celles qui permettraient aux autorités compétentes d'agir rapidement pour obtenir des éléments de preuve et saisir des avoirs faciles à transférer ; les éléments de preuve légalement recueillis par les autorités d'un Etat membre devraient être recevables devant les juridictions des autres Etats membres, compte tenu des règles qui y sont applicables.

54 37. Le Conseil européen demande au Conseil et à la Commission d'adopter, d'ici décembre 2000, un programme de mesures destinées à mettre en œuvre le principe de reconnaissance mutuelle. Dans le cadre de ce programme, des travaux devraient aussi être entamés sur le titre exécutoire européen et sur les aspects du droit procédural 
pour lesquels la fixation de normes minimales communes est considérée comme nécessaire pour faciliter l'application du principe de reconnaissance mutuelle, dans le respect des principes fondamentaux du droit des Etats membres.

VII. Convergence accrue dans le domaine du droit civil

38. Le Conseil européen invite le Conseil et la Commission à élaborer de nouvelles dispositions de droit procédural dans les affaires transfrontières, concernant, en particulier, les éléments qui contribuent à faciliter la coopération judiciaire et à améliorer l'accès au droit, notamment en matière de mesures provisoires, d'obtention des preuves, d'injonctions de payer et de délais.

39. En ce qui concerne le droit matériel, une étude générale doit être réalisée sur la nécessité de rapprocher les législations des Etats membres en matière civile afin d'éliminer les obstacles au bon déroulement des procédures civiles. Le Conseil devra faire rapport d'ici 2001.

\section{LA LUTTE CONTRE LA CRIMINALITE A L'ECHELLE DE L'UNION}

40. Le Conseil européen est fermement décidé à renforcer la lutte contre les formes graves de criminalité organisée et transnationale. Un niveau élevé de sécurité dans l'espace de liberté, de sécurité et de justice suppose une approche efficace et globale de la lutte contre toutes les formes de criminalité. Il faut parvenir à mettre en place de manière équilibrée à l'échelle de l'Union des mesures de lutte contre la criminalité tout en protégeant la liberté des particuliers et des opérateurs économiques et les droits que leur reconnaît la loi.

VIII. Prévention de la criminalité au niveau de l'Union

41. Le Conseil européen demande l'intégration des aspects liés à la prévention dans les actions de lutte contre la criminalité et le développement des programmes nationaux de prévention de la criminalité. Des priorités communes doivent être dégagées et définies en matière de prévention de la criminalité, dans le cadre de la politique extérieure et intérieure de l'Union, et elles devront être prises en compte lors de l'élaboration de nouvelles dispositions législatives.

42. Il convient de développer l'échange des meilleures pratiques, de renforcer le réseau des autorités nationales compétentes en matière de prévention de la criminalité ainsi que la coopération entre les organismes nationaux spécialisés dans ce domaine, et d'étudier à cette fin la possibilité d'un programme qui serait financé par la Communauté. Cette coopération pourrait avoir avant tout pour priorités la délinquance chez les jeunes, la criminalité urbaine et celle liée à la drogue.

IX. Intensifier la coopération dans la lutte contre la criminalité

43. La coopération entre les autorités des Etats membres, lors d'enquêtes sur des activités criminelles transfrontières dans un Etat membre, doit être la plus fructueuse possible. Le Conseil européen demande que les équipes communes d'enquêtes prévues par le traité soient mises sur pied sans délai, dans un premier temps, pour lutter contre le trafic de drogue et la traite des êtres humains, ainsi que contre le terrorisme. Les règles qui seront établies à cet égard devront permettre à des représentants d'Europol de participer, le cas échéant, à ces équipes à titre de soutien.

44. Le Conseil européen demande que soit créée une structure de liaison opérationnelle au sein de laquelle les responsables des services de police européens échangeraient, en coopération avec Europol, expériences, meilleures pratiques et informations sur les 
tendances de la criminalité transfrontière, et contribueraient à l'organisation des opérations.

45. Europol joue un rôle clé de soutien dans la prévention de la criminalité, ainsi que pour l'analyse et les enquêtes criminelles à l'échelle de l'Union. Le Conseil européen invite le Conseil à doter Europol de l'appui et des moyens nécessaires à son action. Europol devrait voir son rôle renforcé prochainement par le fait qu'il recevra des données opérationnelles des Etats membres et qu'il sera habilité à demander aux Etats membres d'engager, de mener ou de coordonner des enquêtes ou de créer des équipes communes d'enquête dans certains domaines de la criminalité, en respectant les systèmes de contrôle par les tribunaux dans les Etats membres.

46. Afin de renforcer la lutte contre les formes graves de criminalité organisée, le Conseil européen a décidé la création d'une unité (Eurojust) composée de procureurs, de magistrats ou d'officiers de police ayant des compétences équivalentes détachés par chaque Etat membre conformément à son système juridique. Eurojust aura pour mission de contribuer à une bonne coordination entre les autorités nationales chargées des poursuites et d'apporter son concours dans les enquêtes relatives aux affaires de criminalité organisée, notamment sur la base de l'analyse effectuée par Europol ; cette unité devra aussi coopérer étroitement avec le Réseau judiciaire européen afin, notamment, de simplifier l'exécution des commissions rogatoires. Le Conseil européen demande au Conseil d'adopter l'instrument juridique nécessaire avant la fin de l'année 2001.

47. Une académie européenne de police doit être créée pour former les hauts responsables des services de police. Elle consistera dans un premier temps en un réseau d'instituts nationaux de formation. Elle devrait également être accessible aux responsables des pays candidats.

48. Sans préjudice des domaines plus larges envisagés dans le traité d'Amsterdam et le plan d'action de Vienne, le Conseil européen estime que, en ce qui concerne le droit pénal national, les efforts visant à trouver un accord sur des définitions, des incriminations et des sanctions communes doivent porter essentiellement, dans un premier temps, sur un nombre limité de secteurs revêtant une importance particulière, tels que la criminalité financière (blanchiment d'argent, corruption, contrefaçon de l'euro), le trafic de drogue, la traite des êtres humains, notamment l'exploitation des femmes, l'exploitation sexuelle des enfants, la criminalité utilisant les technologies avancées et la criminalité au détriment de l'environnement.

49. Les formes graves de criminalité ont de plus en plus d'incidences en matière de taxes et de droits. Le Conseil européen engage donc les Etats membres à fournir sans réserve l'entraide judiciaire pour les enquêtes et les poursuites concernant ce type de criminalité.

71 50. Le Conseil européen insiste sur l'importance d'une approche globale dans la lutte contre la drogue. Il demande au Conseil d'adopter la Stratégie européenne de lutte contre la drogue pour la période 2000-2004 avant la réunion du Conseil européen d'Helsinki.

X. Action spécifique de lutte contre le blanchiment d'argent

73 51. Le blanchiment d'argent est au cœur même de la criminalité organisée. Il faut l'éradiquer partout où il existe. Le Conseil européen est déterminé à veiller à ce que 
soient adoptées des mesures concrètes pour dépister, geler, saisir et confisquer les produits du crime.

52. Les Etats membres sont invités instamment à mettre en œuvre intégralement, y compris dans tous leurs territoires dépendants, les dispositions de la directive sur le blanchiment d'argent, de la convention de Strasbourg de 1990 et les recommandations du Groupe d'action financière sur le blanchiment des capitaux.

53. Le Conseil européen engage le Conseil et le Parlement européen à adopter dès que possible le projet de modification de la directive sur le blanchiment d'argent, récemment proposé par la Commission.

54. Dans le respect des dispositions en matière de protection des données, il convient d'améliorer la transparence des transactions financières et de la provenance du capital des sociétés et d'accélérer l'échange d'informations sur les transactions suspectes entre les cellules de renseignement financier existantes. Quelles que soient les dispositions en matière de confidentialité applicables aux activités bancaires et aux autres activités commerciales, les autorités judiciaires et les cellules de renseignement financier doivent être habilitées, sous le contrôle des tribunaux, à recevoir des informations si celles-ci sont nécessaires dans le cadre d'enquêtes sur le blanchiment d'argent. Le Conseil européen demande au Conseil d'adopter les dispositions nécessaires à cet effet.

55. Le Conseil européen recommande le rapprochement des dispositions de droit et de procédure en matière pénale sur le blanchiment d'argent (notamment en matière de dépistage, de gel et de confiscation d'avoirs). Le champ des activités criminelles constitutives d'infractions principales, dans le domaine du blanchiment d'argent, doit être uniforme et suffisamment large dans tous les Etats membres.

56. Le Conseil européen invite le Conseil à étendre la compétence d'Europol au blanchiment d'argent en général, quel que soit le type d'infraction à l'origine des produits blanchis.

57. Des normes communes doivent être élaborées afin d'empêcher le recours à des sociétés et des entités immatriculées hors du territoire de l'Union pour dissimuler et blanchir le produit d'activités criminelles. L'Union et les Etats membres doivent conclure des accords avec des centres offshore de pays tiers afin d'assurer une coopération efficace et transparente en matière d'entraide judiciaire, conformément aux recommandations formulées à cet égard par le Groupe d'action financière sur le blanchiment de capitaux.

58. La Commission est invitée à établir un rapport recensant les dispositions des législations nationales dans les domaines de la banque, de la finance et des sociétés qui font obstacle à la coopération internationale. Le Conseil est invité à tirer les conclusions nécessaires sur la base de ce rapport.

D. UNE ACTION EXTERIEURE PLUS FORTE

82 59. Le Conseil européen souligne que toutes les compétences et tous les instruments dont dispose l'Union, notamment en matière de relations extérieures, doivent être utilisées de manière intégrée et cohérente pour établir l'espace de liberté, de sécurité et de justice. Les questions de justice et d'affaires intérieures doivent être intégrées dans la définition et la mise en œuvre d'autres politiques et actions de l'Union.

60. Il doit être pleinement fait usage des nouvelles possibilités offertes par le traité d'Amsterdam en matière d'action extérieure et, en particulier, des stratégies 
communes ainsi que des accords conclus par la Communauté et des accords basés sur l'article 38 du traité UE.

84 61. Il convient de définir clairement des priorités, des objectifs politiques et des mesures pour l'action extérieure de l'Union dans le domaine de la justice et des affaires intérieures. Le Conseil doit, en étroite collaboration avec la Commission, élaborer des recommandations spécifiques concernant les objectifs et les mesures relatives à cette action extérieure, notamment pour ce qui est des structures de travail, avant le Conseil européen de juin 2000.

62. Le Conseil européen appuie la coopération régionale entre les Etats membres et les pays tiers limitrophes de l'Union en matière de lutte contre la criminalité organisée. A cet égard, il prend acte avec satisfaction des résultats concrets et pratiques obtenus par les pays riverains de la Baltique. Le Conseil européen attache une importance particulière à la coopération et au développement régionaux dans la région des Balkans. L'Union européenne se félicite qu'une Conférence européenne sur le développement et la sécurité dans la région de la mer Adriatique et de la mer Ionienne soit organisée par le gouvernement italien en Italie au cours du premier semestre de l'année 2000 et a l'intention d'y participer. Cette initiative apportera une contribution utile dans le cadre du Pacte de stabilité pour l'Europe du Sud-Est. (...)

\section{INDEX}

Mots-clés : textes et documents officiels, construction européenne 